\title{
Origin of universality in the onset of superdiffusion in Lévy walks
}

\author{
Asaf Miron 10 \\ Department of Physics of Complex Systems, Weizmann Institute of Science, Rehovot 7610001, Israel
}

(Received 22 June 2020; accepted 3 August 2020; published 19 August 2020)

\begin{abstract}
Superdiffusion arises when complicated, correlated, and noisy motion at the microscopic scale conspires to yield peculiar dynamics at the macroscopic scale. It ubiquitously appears in a variety of scenarios, spanning a broad range of scientific disciplines. The approach of superdiffusive systems towards their long-time, asymptotic behavior was recently studied using the Lévy walk of order $1<\beta<2$, revealing a universal transition at the critical $\beta_{c}=3 / 2$. Here, we investigate the origin of this transition and identify two crucial ingredients: a finite velocity which couples the walker's position to time and a corresponding transition in the fluctuations of the number of walks $n$ completed by the walker at time $t$.
\end{abstract}

DOI: 10.1103/PhysRevResearch.2.032042

Introduction. Diffusion effectively models the dynamics of many physical systems. Its hallmark property, a linear increase of the mean-square displacement (MSD) with time, famously describes the stagnant motion of a grain of pollen tumbling about in a glass of water [1]. Yet there is an ever-growing list of "superdiffusive" phenomena that fall well outside the paradigm of simple diffusion, in which perturbations propagate faster than diffusion. Notable examples include the dynamics of turbulent systems [2], spreading of perturbations and associated anomalous transport [3-8], tagged particle dynamics in disordered media $[9,10]$, evolution of trapped ions and atoms in optical lattices [11-13], and even the behavior exhibited by living matter [14-19].

The Lévy walk is a canonical model for superdiffusion. In 1D, it describes a "walker" evolving in a series of independent "walks." At the start of each walk, the walker randomly draws a "walk time" $\tau$ and a direction \pm 1 along which it moves with velocity $\pm v$ for the duration of the walk. Superdiffusion arises when the walk-time distribution $\phi(\tau)$ features a heavy tail that scales as $\propto \tau^{-1-\beta}$ for large $\tau$, with $1<\beta<2$ called the "order" of the Lévy walk. While the mean walk time $\langle\tau\rangle$ remains finite, naively suggesting a diffusive motion consisting of short walks of duration $\propto\langle\tau\rangle$, the divergence of $\phi(\tau)$ 's second moment $\left\langle\tau^{2}\right\rangle$ signals the existence of unbounded fluctuations that occasionally lead the walker on very long walks, ultimately yielding superdiffusive dynamics. For comparison, when $\beta>2$, the second moment $\left\langle\tau^{2}\right\rangle$ remains finite and simple diffusion is recovered [20]. The finite speed $v>0$ couples the walker's position to time, prevents it from traveling a distance greater than $v t$ over a time $t$, and guarantees the locality of its dynamics. This seemingly innocent property significantly complicates the Lévy walk's

Published by the American Physical Society under the terms of the Creative Commons Attribution 4.0 International license. Further distribution of this work must maintain attribution to the author(s) and the published article's title, journal citation, and DOI. analysis compared to other superdiffusive models, such as the Lévy flight and the continuous-time random walk (CTRW), which are often easier to analyze but have also been criticized for their nonlocal dynamics [20,21].

The approach of superdiffusive systems towards their longtime asymptotic form was recently studied using the Lévy walk of order $1<\beta<2$ [22]. The walker's probability density $P(x, t)$ was studied for large $t$ yet beyond the known asymptotic solution $P_{0}(x, t)=t^{-1 / \beta} f\left(t^{-1 / \beta} x\right)$ [20,22]. This approach, captured by the leading correction to $P_{0}(x, t)$ as $t \rightarrow \infty$, was shown to transition at $\beta_{c}=3 / 2$ between a diffusive scaling $|x| \propto t^{1 / 2}$ for $\beta>\beta_{c}$ and a superdiffusive scaling $|x| \propto t^{1 /(2 \beta-1)}$ for $\beta<\beta_{c}$. This transition [23] is regarded universal as it was shown to be insensitive to $\phi(\tau)$ 's short-time behavior, depending only on its heavy tail $\propto \tau^{-1-\beta}$ [22]. Indeed, recent results concerning anomalous transport in a class of 1D systems [24] modeled by a Lévy walk of order $\beta=5 / 3[3,8,25]$ are consistent with the diffusive correction predicted in Lévy walks for $\beta>\beta_{c}$ [22]. This raises the exciting possibility that Lévy walks may remarkably remain a valid description of superdiffusive phenomena, even beyond the asymptotic limit. Elucidating how superdiffusive systems approach their asymptotic behavior thus carries both a theoretical appeal as well as concrete consequences for experimental and numerical investigations of superdiffusive phenomena, which are inherently limited to finite space and time [22]. Still, one pressing question remains unanswered: What is the origin of this transition?

In this Rapid Communication, we investigate the mechanism responsible for the universal transition observed in the onset of superdiffusion in Lévy walks [22]. We find it to be twofold, consisting of the finite speed $v$ which couples the walker's position to time and a corresponding transition at $\beta_{c}=3 / 2$ in the fluctuations $\left\langle\Delta n_{t}^{2}\right\rangle \equiv\left\langle n_{t}^{2}\right\rangle-\left\langle n_{t}\right\rangle^{2}$ of the number of walks $n$ completed by the walker at time $t$. At large $t$ these become $\left\langle\Delta n_{t}^{2}\right\rangle \cong\left\langle\Delta n_{t}^{2}\right\rangle_{0}+\left\langle\delta n_{t}^{2}\right\rangle$ with the asymptotic fluctuations $\left\langle\Delta n_{t}^{2}\right\rangle_{0}=\kappa_{0} t^{3-\beta}$ interpolating between a ballistic scaling for $\beta=1$ and a diffusive scaling for $\beta=2$. Yet going beyond the asymptotic limit reveals a transition 
in the preasymptotic fluctuations $\left\langle\delta n_{t}^{2}\right\rangle \cong \kappa_{1} t+\kappa_{2} t^{4-2 \beta}$. For $\beta>\beta_{c}$ one finds $\left\langle\delta n_{t}^{2}\right\rangle \propto t$, as expected in simple diffusion (i.e., for $\beta>2$ ). For $\beta<\beta_{c}$, however, one instead finds a superdiffusive scaling $\left\langle\delta n_{t}^{2}\right\rangle \propto t^{4-2 \beta}$. The transition in $\left\langle\delta n_{t}^{2}\right\rangle$ enters the Lévy walker's position through its coupling to time via $v$, inducing a corresponding transition in the onset of superdiffusion in the Lévy walk propagator, hereby causally tying the two transitions. Yet, clearly, these fluctuations may only affect models with local dynamics, where the distance traveled by the particle is proportional to the traveling time. As such, we complete the picture by explicitly demonstrating the absence of a transition in the onset of superdiffusion in the Lévy flight and CTRW models, where the lack of a coupling between the particle's position and time yields nonlocal dynamics. Besides explaining the onset of superdiffusion, the transition in $\left\langle\delta n_{t}^{2}\right\rangle$ also provides a tractable observable that can be used to probe the value of $\beta$ by tracking the number of typical "ballistic" excursions in superdiffusive experimental and simulation data.

Evidently, the observable $\left\langle\Delta n_{t}^{2}\right\rangle$ also carries significant interest in the context of "renewal processes" [26,27], which describe physical scenarios where the time intervals between events are modeled as independent and identically distributed random variables. When these intervals happen to be drawn from a heavy-tailed distribution, with the same tail behavior $\alpha \tau^{-1-\beta}$ as considered above, the fluctuations in the number of events are analogous to the number of walks performed by the Lévy walker, similarly spreading as $\left\langle\Delta n_{t}^{2}\right\rangle_{0} \propto t^{3-\beta}$ for asymptotically long times. Such behavior has been linked to blinking quantum dots [28], as well as in the diffusion of particles in polymer networks [29] and on cell membranes [30]. The interest in the transition in $\left\langle\delta n_{t}^{2}\right\rangle$, which should similarly appear in such processes for $1<\beta<2$, is thus expected to extend far beyond the context of the onset of superdiffusion in Lévy walks.

Model. The 1D Lévy walk of order $\beta$ describes the evolution of a walker along an infinite line in a series of uncorrelated walks $[20,31]$. In each walk, the walker randomly draws a direction \pm 1 and a walk time $\tau$ from the walk-time distribution $\phi(\tau)$, and proceeds to walk along the chosen direction with velocity $\pm v$ until $\tau$ expires and the process repeats. These dynamics become superdiffusive when $\phi(\tau)$ features a heavy tail that scales as $\propto \tau^{-1-\beta}$ for large $\tau$ and $1<\beta<2$. In what follows we shall consider the convenient choice

$$
\phi(\tau)=\beta t_{0}^{\beta} \theta\left[\tau-t_{0}\right] \tau^{-(1+\beta)} \text { for } 1<\beta<2,
$$

where the step function $\theta[x]$ keeps $\phi(\tau)$ normalizable on $\tau \in$ $[0, \infty)$ by imposing a cutoff at the minimal walk time $t_{0}$. For simplicity, however, we shall henceforth set $t_{0}=1$, effectively rendering $\tau$ to be a dimensionless time.

To study the fluctuations in the number of steps $n$ completed by the walker at time $t$, we generalize the Lévy walk model [20] and formulate self-consistent equations for two quantities: the density per unit time $v_{n}(x, t)$ of walkers leaving position $x$ at time $t$ after completing $n$ walks and the density $P_{n}(x, t)$ of walkers at position $x$ at time $t$ during the $n^{\text {th }}$ walk.

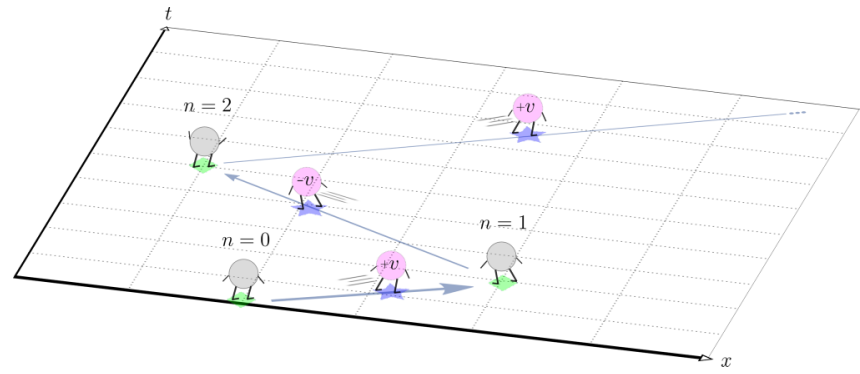

FIG. 1. A schematic illustration of the first few steps of the Lévy walk dynamics. Green diamonds mark the walker's position after completing its $n^{\text {th }}$ walk, as described by $v_{n}(x, t)$ in Eq. (2). Purple stars mark the walker's position during its $n^{\text {th }}$ walk, as described by $P_{n}(x, t)$ in Eq. (3).

The equations for $v_{n}(x, t)$ and its initial condition are

$$
\begin{aligned}
v_{n+1}(x, t)= & \frac{1}{2} \int_{-\infty}^{\infty} \mathbf{d} y \int_{0}^{t} \mathbf{d} \tau \delta[|y|-v \tau] \\
& \times \phi(\tau) v_{n}(x-y, t-\tau) \text { and } v_{0}(x, t)=\delta(x) \delta(t) .
\end{aligned}
$$

The right-hand side accounts for contributions to $v_{n+1}(x, t)$ from walkers that have just completed $n$ steps and are located at $x-y$ at time $t-\tau$. The equation for $P_{n}(x, t)$ is given by [32]

$$
P_{n}(x, t)=\frac{1}{2} \int_{-\infty}^{\infty} \mathbf{d} y \int_{0}^{t} \mathbf{d} \tau \delta[|y|-v \tau] \psi(\tau) v_{n}(x-y, t-\tau),
$$

describing contributions to $P_{n}(x, t)$ from walkers beginning their $n^{\text {th }}$ walk at position $x-y$ at time $t-\tau$ and drawing a walk time greater than $\tau$, which occurs with probability

$$
\psi(\tau)=\int_{\tau}^{\infty} \mathbf{d} u \phi(u)
$$

These walkers pass through $x$ at time $t$, before the walk's duration had expired, and then continue walking. Since we consider a $\delta(x)$ initial condition and the number of walkers is conserved, $P_{n}(x, t)$ is hereafter referred to as the "generalized propagator" and may be thought of as a probability density of a single walker. The model's dynamics and the differences between $v_{n}(x, t)$ and $P_{n}(x, t)$ are illustrated in Fig. 1.

Main results. The universal transition in the onset of superdiffusion in Lévy walks [22] is shown to trace back to a corresponding transition in the fluctuations $\left\langle\Delta n_{t}^{2}\right\rangle$ of the number of walks $n$ completed by the walker at time $t$. These fluctuations, and the entailing transition, then enter the walker's position through its coupling to time via $v$, inducing a corresponding transition in the Lévy walk propagator. We first compute the generalized propagator $P_{n}(x, t)$, from which we derive an exact expression for the walk-number distribution $\tilde{Q}_{n}(s)$ in Laplace space in Eq. (11). The real-time distribution $Q_{n}(t)$ is verified against direct numerical simulations in Fig. 2. We next explicitly evaluate the large- $t$ walk-number 


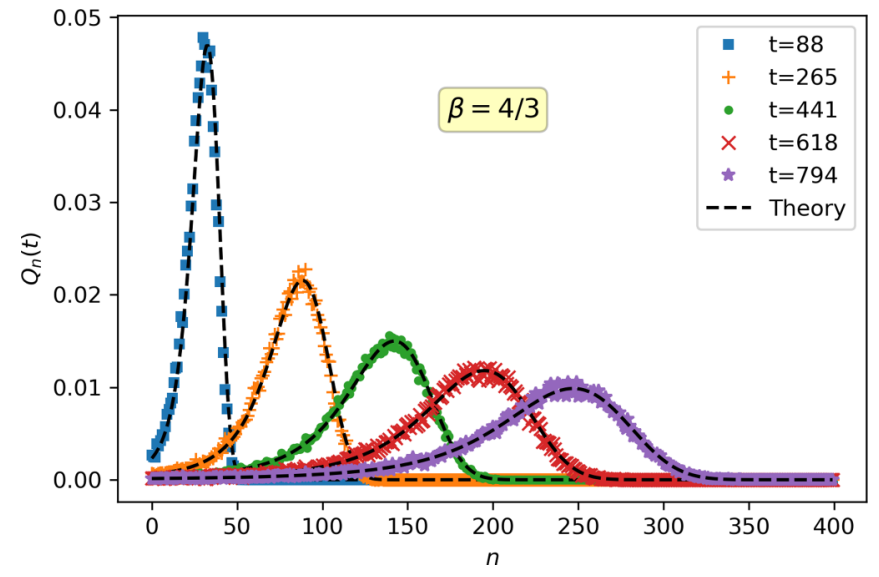

FIG. 2. The walk-number distribution $Q_{n}(t)$ for $\beta=\beta_{c}$ vs the number of steps $n$. Markers depict the simulated walk-number distribution while the dashed black lines represent the numerical inverse Laplace transform of $\tilde{Q}_{n}(s)$ in Eq. (11) at different times. The temporal growth of the distribution's width is described by $\left\langle\Delta n_{t}^{2}\right\rangle$.

fluctuations in Eq. (17), finding

$$
\left\langle\Delta n_{t}^{2}\right\rangle \cong\left\langle\Delta n_{t}^{2}\right\rangle_{0}+\left\langle\delta n_{t}^{2}\right\rangle
$$

where $\left\langle\Delta n_{t}^{2}\right\rangle_{0}=\kappa_{0} t^{3-\beta}$ describes the asymptotic behavior while $\left\langle\delta n_{t}^{2}\right\rangle=\kappa_{1} t+\kappa_{2} t^{4-2 \beta}$ accounts for the preasymptotic fluctuations that, as shown in Fig. 3, undergo a transition at $\beta_{c}=3 / 2$ with $\kappa_{0}, \kappa_{1}$, and $\kappa_{2}$ given in Eq. (18). Specifically, for $\beta>\beta_{c}$ we recover diffusive fluctuations $\left\langle\delta n_{t}^{2}\right\rangle \propto t$, whereas for $\beta<\beta_{c}$ the preasymptotic fluctuations instead grow superdiffusively as $\left\langle\delta n_{t}^{2}\right\rangle \propto t^{4-2 \beta}$. Clearly, fluctuations in $n$ may affect the propagator only if the distance traveled by the particle is proportional to the traveling duration. Nevertheless, we complete the picture by demonstrating the absence of a transition in the onset of superdiffusion in the Lévy flight and CTRW models, where the particle's position is not coupled to time. Consequently, although a transition in $\left\langle\delta n_{t}^{2}\right\rangle$ is found in the CTRW model, it fails to induce a corresponding transition in the onset of superdiffusion.

Generalized propagator. Applying a Fourier-Laplace transform to Eq. (2) for $v_{n}(x, t)$ yields

$$
\begin{aligned}
\tilde{v}_{n+1}(k, s)= & \frac{1}{2} \int_{0}^{\infty} \mathbf{d} t e^{-s t} \int_{0}^{t} \mathbf{d} \tau \phi(\tau) \\
& \times\left(e^{i v k \tau}+e^{-i v k \tau}\right) \hat{v}_{n}(k, t-\tau),
\end{aligned}
$$

where we denote the Fourier transform by $\hat{f}(k, t)=$ $\int_{-\infty}^{\infty} \mathbf{d} x e^{-i k x} f(x, t)$ and the Laplace transform by $\tilde{f}(k, s)=$ $\int_{0}^{\infty} \mathbf{d} t e^{-s t} \hat{f}(k, t)$. Interchanging the order of integration of $t$ and $\tau$, i.e., $\int_{0}^{\infty} \mathbf{d} t \int_{0}^{t} \mathbf{d} \tau \rightarrow \int_{0}^{\infty} \mathbf{d} \tau \int_{\tau}^{\infty} \mathbf{d} t$, lets us reduce Eq. (6) to

$$
\tilde{v}_{n+1}(k, s)=\frac{1}{2}[\tilde{\phi}(s-i v k)+\tilde{\phi}(s+i v k)] \tilde{v}_{n}(k, s) .
$$

Solving the $n$ dependence in Eq. (7) subject to the initial condition in Eq. (2) gives

$$
\tilde{v}_{n}(k, s)=\left(\frac{1}{2}[\tilde{\phi}(s-i v k)+\tilde{\phi}(s+i v k)]\right)^{n} .
$$

Applying the same approach to Eq. (3) for $P_{n}(x, t)$ leads to

$$
\tilde{P}_{n}(k, s)=\frac{1}{2} \tilde{v}_{n}(k, s)[\tilde{\psi}(s-i v k)+\tilde{\psi}(s+i v k)] .
$$

The formal solution for $\tilde{P}_{n}(k, s)$ is obtained by combining Eqs. (8) and (9) into

$$
\tilde{P}_{n}(k, s)=\frac{\tilde{\psi}(s-i v k)+\tilde{\psi}(s+i v k)}{2^{n+1}[\tilde{\phi}(s-i v k)+\tilde{\phi}(s+i v k)]^{-n}} .
$$

The generalized Lévy walk propagator $\tilde{P}_{n}(k, s)$ in Eq. (10) is consistent with the known Lévy walk propagator $\tilde{P}^{\mathrm{LW}}(k, s)=\frac{\tilde{\psi}(s-i v k)+\tilde{\psi}(s+i v k)}{2-\tilde{\phi}(s-i v k)-\tilde{\phi}(s+i v k)}$ [20], which is immediately recovered when summing $\tilde{P}_{n}(k, s)$ over $n$. For large $t$ and small $|k|, \tilde{P}^{\mathrm{LW}}(k, s)$ was shown in Ref. [22] to asymptotically approach $\hat{P}^{\mathrm{LW}}(q, t) \cong e^{-t I(q)}$, where $I(q)=$ $(1-\operatorname{Re}[\hat{\phi}(q)]) / \partial_{q} \operatorname{Im}[\hat{\phi}(q)]$ and $q=v k$. There, this nontrivial functional dependence of $I(q)$ on $\hat{\phi}(q)$ stems from the spatiotemporal coupling by $v$, as dictated by the distribution
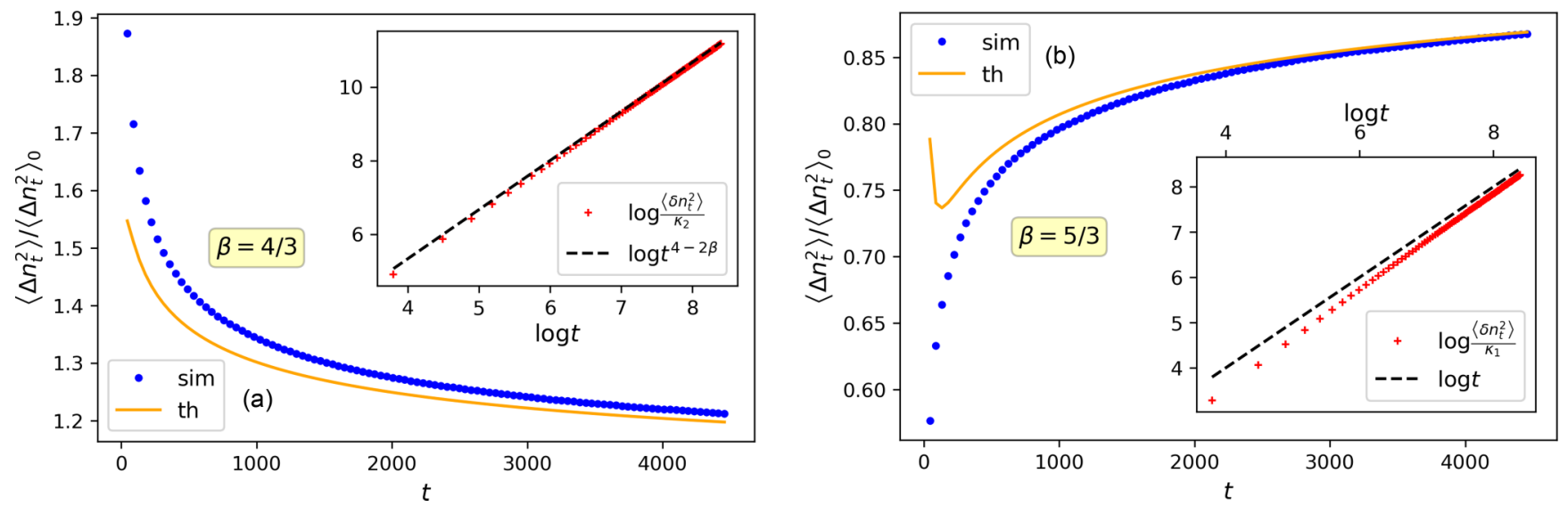

FIG. 3. The large- $t$ fluctuations $\left\langle\Delta n_{t}^{2}\right\rangle$ over the asymptotic fluctuations $\left\langle\Delta n_{t}^{2}\right\rangle_{0}$ of Eq. (17) vs $t$. As $t \rightarrow \infty$, this ratio approaches unity from above for $\beta<\beta_{c}$ in (a) and from below for $\beta>\beta_{c}$ in (b). Blue circles depict the simulated $\left\langle\Delta n_{t}^{2}\right\rangle$ while the solid orange curve depicts its theoretical expression of Eq. (17). The insets show a logarithmic plot of the preasymptotic fluctuations $\left\langle\delta n_{t}^{2}\right\rangle=\kappa_{1} t+\kappa_{2} t^{4-2 \beta}$ vs $t$, where $\left\langle\delta n_{t}^{2}\right\rangle$ is obtained by subtracting the theoretical $\left\langle\Delta n_{t}^{2}\right\rangle_{0}$ from the simulated fluctuations $\left\langle\Delta n_{t}^{2}\right\rangle$. Specifically, (a) depicts $\log \left\langle\delta n_{t}^{2}\right\rangle / \kappa_{2}$ by red crosses and $(4-2 \beta) \log t$ by a black dashed curve for $\beta=4 / 3<\beta_{c}$ while (b) uses the same scheme to depict $\log \left\langle\delta n_{t}^{2}\right\rangle / \kappa_{1}$ and $\log t$ for $\beta=5 / 3>\beta_{c}$. 
$\tilde{\phi}(s \pm i v k)$, and ultimately yields the transition in the onset of superdiffusion in Lévy walks. While uncovering the origin of this transition, we shall see that the coupling between the walk's position and time is, in fact, a simple, natural, and intuitive mechanism which serves to intertwine the walk's position with the corresponding transition in the walk-number fluctuations.

Walk-number fluctuations. Our next task is to evaluate the walk-number fluctuations $\left\langle\Delta n_{t}^{2}\right\rangle$. To this end, we first marginalize the generalized propagator $\tilde{P}_{n}(k, s)$ over the walker's position by setting $k=0$. This, along with the Laplace transform $\tilde{\psi}(s)=s^{-1}[1-\tilde{\phi}(s)]$ of $\psi(\tau)$ in Eq. (4), yields the Laplace-transformed walk-number distribution

$$
\tilde{Q}_{n}(s) \equiv \tilde{P}_{n}(k=0, s)=s^{-1}[1-\tilde{\phi}(s)] \tilde{\phi}(s)^{n} .
$$

While we here derive it from the generalized propagator $\tilde{P}_{n}(k, s)$, we stress that $\tilde{Q}_{n}(s)$ is a more fundamental quantity that can be obtained without considering the Lévy walker's spatial behavior [33]. To proceed, we introduce the Laplacespace moment generating function

$$
g(s ; \lambda)=\sum_{n=0}^{\infty} \lambda^{n} \tilde{Q}_{n}(s)=\frac{1-\tilde{\phi}(s)}{s[1-\lambda \tilde{\phi}(s)]},
$$

from which we derive

$$
\left\langle\tilde{n}_{s}\right\rangle=\frac{\tilde{\phi}(s)}{s[1-\tilde{\phi}(s)]} \text { and }\left\langle\tilde{n}_{s}^{2}\right\rangle=\frac{\tilde{\phi}(s)[1+\tilde{\phi}(s)]}{s[1-\tilde{\phi}(s)]^{2}},
$$

noting that $\left\langle\tilde{n}_{s}^{m}\right\rangle=\int_{0}^{\infty} \mathbf{d} t e^{-s t}\left\langle n_{t}^{m}\right\rangle$ is the Laplace transform of the $m^{\text {th }}$ moment $\left\langle n_{t}^{m}\right\rangle$.

To keep our discussion as general as possible, let us consider a generic walk-time distribution $\phi(\tau)$ with an analytic short-time behavior and a tail which scales as $\propto \tau^{-1-\beta}$ for large $\tau$. The Laplace transform $\tilde{\phi}(s)=\int_{0}^{\infty} \mathbf{d} t e^{-s t} \phi(t)$ of such a general distribution is given by

$$
\tilde{\phi}(s)=s^{\beta} \sum_{r=0}^{\infty} d_{r} s^{r}+\sum_{m=0}^{\infty} c_{m} s^{m} .
$$

The singular terms $s^{\beta} \sum_{r=0}^{\infty} d_{r} s^{r}$ account for the distribution's tail and are responsible for the divergence of the second and higher moments, while the analytic series $\sum_{m=0}^{\infty} c_{m} s^{m}$ captures its short-time behavior. The coefficients $\left\{c_{m}\right\}_{m=0}^{\infty}$ and $\left\{d_{r}\right\}_{r=0}^{\infty}$ may be uniquely determined for any such walk-time distribution $\phi(\tau)$, including the choice in Eq. (1) which was used in Figs. 2 and 3 but also for other choices [33]. We proceed to analyze the large- $t$ behavior of $\left\langle\Delta n_{t}^{2}\right\rangle$ by first obtaining the small-s (i.e., large- $t$ ) behavior of $\left\langle\tilde{n}_{s}\right\rangle$ and $\left\langle\tilde{n}_{s}^{2}\right\rangle$ in Eq. (13) and then taking the inverse Laplace transform [33]. We find

$$
\left\langle n_{t}\right\rangle \approx-\frac{t}{c_{1}}+\frac{d_{0} t^{2-\beta}}{\Gamma[3-\beta] c_{1}^{2}}+\frac{c_{2}-c_{1}^{2}}{c_{1}^{2}},
$$

and

$$
\left\langle n_{t}^{2}\right\rangle \approx \frac{t^{2}}{c_{1}^{2}}-\frac{4 d_{0} t^{3-\beta}}{\Gamma[4-\beta] c_{1}^{3}}+\frac{\left(3 c_{1}^{2}-4 c_{2}\right) t}{c_{1}^{3}}+\frac{6 d_{0} t^{4-2 \beta}}{\Gamma[5-2 \beta] c_{1}^{4}},
$$

where higher-order terms are neglected and the normalization condition $c_{0}=1$ is used. The leading large- $t$ behavior of
$\left\langle\Delta n_{t}^{2}\right\rangle \approx\left\langle\Delta n_{t}^{2}\right\rangle_{0}+\left\langle\delta n_{t}^{2}\right\rangle$ in Eq. (5) is thus

$$
\left\langle\Delta n_{t}^{2}\right\rangle_{0}=\kappa_{0} t^{3-\beta} \text { and }\left\langle\delta n_{t}^{2}\right\rangle=\kappa_{1} t+\kappa_{2} t^{4-2 \beta} \text {, }
$$

with the coefficients $\kappa_{0}, \kappa_{1}$, and $\kappa_{2}$ given by

$$
\kappa_{0}=\frac{2(1-\beta) d_{0}}{\Gamma[4-\beta] c_{1}^{3}}, \quad \kappa_{1}=\frac{c_{1}^{2}-2 c_{2}}{c_{1}^{3}},
$$

and

$$
\kappa_{2}=\frac{d_{0}}{c_{1}^{4}}\left(\frac{6}{\Gamma[5-2 \beta]}-\frac{1}{\Gamma[3-\beta]^{2}}\right) .
$$

For the choice of $\phi(\tau)$ in Eq. (1), one finds $c_{1}=-\frac{\beta}{\beta-1}$, $d_{0}=-\Gamma[1-\beta]$, and $c_{2}=-\frac{\beta}{2(2-\beta)}$ [33]. These walk-number fluctuations enter the Lévy walk propagator since the distance traveled by the walker is proportional to its traveling time. As such, the transition in the preasymptotic fluctuations $\left\langle\delta n_{t}^{2}\right\rangle$ induces a corresponding transition in the onset of superdiffusion.

The Lévy flight and CTRW models. We finally demonstrate the absence of a transition in the onset of superdiffusion in the Lévy flight and CTRW models, where the distance traveled by the particle is not proportional to the traveling time. Moreover, we explicitly show that the CTRW's nonlocal dynamics fail to produce a transition in the onset of superdiffusion, even though the model does exhibit a transition in $\left\langle\delta n_{t}^{2}\right\rangle$. Explicit calculations and details are provided in the Supplemental Material [33].

In each step of the 1D CTRW dynamics, the particle waits a random time $\tau$ and then makes a random jump $\ell$ [20]. Superdiffusion arises when the waiting-time distribution scales as $\omega(\tau) \propto \tau^{-1-\beta}$ for large $\tau$ and has a finite first moment $\langle\tau\rangle$, corresponding to $\beta>1$, while the symmetric jump-distance distribution scales as $g(\ell) \propto|\ell|^{-1-\gamma}$ for large $|\ell|$ and has a diverging second moment $\left\langle\ell^{2}\right\rangle \rightarrow \infty$, corresponding to $1<$ $\gamma<2$. Generalizing the CTRW dynamics to account for the number of steps $n$, as in Eqs. (2) and (3) for the Lévy walk model, one obtains the generalized CTRW propagator

$$
\tilde{P}_{n}^{\mathrm{CTRW}}(k, s)=s^{-1}[1-\tilde{\omega}(s)] \hat{g}(k)^{n} \tilde{\omega}(s)^{n} .
$$

Marginalizing over space gives the walk-number distribution $\tilde{Q}_{n}^{\mathrm{CTRW}}(s)=s^{-1}[1-\tilde{\omega}(s)] \tilde{\omega}(s)^{n}$, which is identical to that obtained in Eq. (11) for the Lévy walk. As such, the same transition arises in the preasymptotic walk-number fluctuations $\left\langle\delta n_{t}^{2}\right\rangle$ at $\beta_{c}$, as in Eq. (17). However, by marginalizing Eq. (19) over $n$ and taking the long-time and large-distance limit, one finds $\tilde{P}^{\mathrm{CTRW}}(k, t) \cong e^{-t\left[\bar{D}_{0}|k|^{\gamma}-\bar{D}_{1} k^{2}+O\left(|k|^{2+\gamma}\right)\right]}$, where $\bar{D}_{0}$ and $\bar{D}_{1}$ depend on the details of $\omega(\tau)$ and $g(\ell)$. Since the leading correction to the asymptotic CTRW propagator $\tilde{P}_{0}^{\mathrm{CTRW}}(k, t)=e^{-\bar{D}_{0} t|k|^{\gamma}}$ is proportional to $\sim k^{2}$ for any $1<$ $\gamma<2$, no transition arises in the onset of superdiffusion.

A similar picture is found in the 1D Lévy flight, which describes a "flier" whose discrete evolution consists of repeatedly drawing a flight distance $\ell$ from the distribution $\xi(\ell)$ and immediately materializing at its new location. Superdiffusion appears when $\xi(\ell)$ 's symmetric tails scale as $\xi(\ell) \propto$ $|\ell|^{-1-\beta}$ for large $|\ell|$ and $1<\beta<2$. The model's discrete evolution is neatly contained within the generalized Lévy walk dynamics of Eqs. (2) and (3) and its known propagator $\hat{P}_{n}^{\mathrm{LF}}(k)=\hat{\phi}(k)^{n}$ is recovered from $\hat{v}_{n}(k, s)$ of Eq. (8) by 
setting $s=0$. For large distances and $n$, it assumes the form $\hat{P}_{n}^{\mathrm{LF}}(k) \cong e^{-n\left[D_{0}|k|^{\beta}-D_{1} k^{2}+O\left(k^{4}\right)\right]}$, where $D_{0}$ and $D_{1}$ depend on the details of $\xi(\ell)$. Again, no transition appears in the onset of superdiffusion.

Conclusions. In this Rapid Communication, we studied the mechanism behind the recently reported universal transition in the onset of superdiffusion in Lévy walks of order $1<$ $\beta<2$. It was shown to be twofold, consisting of the finite speed $v$ which couples the walker's position to time and a corresponding transition in the fluctuations of the number of walks $n$ completed by the walker at time $t$. Generalizing the Lévy walk model to account for the number of walks $n$ allowed us to compute the walk-number distribution and its large- $t$ fluctuations $\left\langle\Delta n_{t}^{2}\right\rangle \cong\left\langle\Delta n_{t}^{2}\right\rangle_{0}+\left\langle\delta n_{t}^{2}\right\rangle$. A transition was demonstrated in the preasymptotic fluctuations $\left\langle\delta n_{t}^{2}\right\rangle=$ $\kappa_{1} t+\kappa_{2} t^{4-2 \beta}$, showing diffusive behavior $\left\langle\delta n_{t}^{2}\right\rangle \propto t$ for $\beta>$ $\beta_{c}$ and superdiffusive behavior $\left\langle\delta n_{t}^{2}\right\rangle \propto t^{4-2 \beta}$ for $\beta<\beta_{c}$. This picture was completed by showing that no transition occurs in the onset of superdiffusion in the Lévy flight and CTRW models, where the particle's position is not coupled to time.

Unlike the full propagator, which is nutritiously hard to obtain from data, the walk-number fluctuations $\left\langle\Delta n_{t}^{2}\right\rangle$ can be readily extracted from the dynamics by tracking the evolution of the number of typical "ballistic" excursions observed in superdiffusive systems. This study shows that this robust and accessible observable can be used to precisely predict which systems are expected to exhibit a transition in the onset of superdiffusion, be they experimental or numerical. However, besides its theoretical value in uncovering the mechanism responsible for the transition in the onset of superdiffusion [22], the transition in $\left\langle\Delta n_{t}^{2}\right\rangle$ can itself be used as a tool for precisely determining the value of $\beta$. This collateral contribution is important since only a few such instruments are currently known, in spite of the well-known and often devastating difficulties posed by finite-time corrections in both experimental and numerical studies of superdiffusive phenomena [3,34-41]. This work joins the efforts detailed in Refs. [8,22,24,39,42,43] of establishing an understanding of the preasymptotic behavior of superdiffusive systems. In this context, it would be very interesting to test these predictions in experimental and numerical systems which are modeled by Lévy walks.

Acknowledgments. I thank David Mukamel for his ongoing encouragement and support, for critically reading this manuscript, and for many helpful discussions. I also thank Julien Cividini and Oren Raz for critically reading this manuscript and for their helpful remarks. This work was supported by a research grant from the Center of Scientific Excellence at the Weizmann Institute of Science.
[1] R. Brown, XXVII. A brief account of microscopical observations made in the months of June, July and August 1827, on the particles contained in the pollen of plants; and on the general existence of active molecules in organic and inorganic bodies, Philos. Mag. 4, 161 (1828).

[2] M. F. Shlesinger, B. J. West, and J. Klafter, Lévy Dynamics of Enhanced Diffusion: Application to Turbulence, Phys. Rev. Lett. 58, 1100 (1987).

[3] P. Cipriani, S. Denisov, and A. Politi, From Anomalous Energy Diffusion to Levy Walks and Heat Conductivity in OneDimensional Systems, Phys. Rev. Lett. 94, 244301 (2005).

[4] V. Zaburdaev, S. Denisov, and P. Hänggi, Perturbation Spreading in Many-Particle Systems: A Random Walk Approach, Phys. Rev. Lett. 106, 180601 (2011).

[5] S. Liu, X. F. Xu, R. G. Xie, G. Zhang, and B. W. Li, Anomalous heat conduction and anomalous diffusion in low dimensional nanoscale systems, Eur. Phys. J. B 85, 337 (2012).

[6] A. Dhar, K. Saito, and B. Derrida, Exact solution of a Lévy walk model for anomalous heat transport, Phys. Rev. E 87, 010103 (2013).

[7] J. Cividini, A. Kundu, A. Miron, and D. Mukamel, Temperature profile and boundary conditions in an anomalous heat transport model, J. Stat. Mech.: Theory Exp. (2017) 013203.

[8] A. Miron, Lévy walks on finite intervals: A step beyond asymptotics, Phys. Rev. E 100, 012106 (2019).

[9] P. Levitz, From Knudsen diffusion to Levy walks, Europhys. Lett. 39, 593 (1997).

[10] D. Brockmann and T. Geisel, Lévy Flights in Inhomogeneous Media, Phys. Rev. Lett. 90, 170601 (2003).

[11] S. Marksteiner, K. Ellinger, and P. Zoller, Anomalous diffusion and Lévy walks in optical lattices, Phys. Rev. A 53, 3409 (1996).
[12] H. Katori, S. Schlipf, and H. Walther, Anomalous Dynamics of a Single Ion in an Optical Lattice, Phys. Rev. Lett. 79, 2221 (1997).

[13] Y. Sagi, M. Brook, I. Almog, and N. Davidson, Observation of Anomalous Diffusion and Fractional Self-Similarity in One Dimension, Phys. Rev. Lett. 108, 093002 (2012).

[14] A. Ott, J. P. Bouchaud, D. Langevin, and W. Urbach, Anomalous Diffusion in "Living Polymers": A Genuine Lévy Flight? Phys. Rev. Lett. 65, 2201 (1990).

[15] S. V. Buldyrev, A. L. Goldberger, S. Havlin, C.-K. Peng, M. Simons, and H. E. Stanley, Generalized Lévy-walk model for DNA nucleotide sequences, Phys. Rev. E 47, 4514 (1993).

[16] A. Upadhyaya, J.-P. Rieu, J. A. Glazier, and Y. Sawada, Anomalous diffusion and non-Gaussian velocity distribution of hydra cells in cellular aggregates, Physica A 293, 549 (2001).

[17] A. M. Reynolds, Current status and future directions of Lévy walk research, Biol. Open 7, bio030106 (2018).

[18] I. Rhee, M. Shin, S. Hong, K. Lee, S. J. Kim, and S. Chong, On the Lévy-walk nature of human mobility, IEEE/ACM Trans. Netw. 19, 630 (2011).

[19] D. A. Raichlen, B. M. Wood, A. D. Gordon, A. Z. P. Mabulla, F. W. Marlowe, and H. Pontzer, Evidence of Lévy walk foraging patterns in human hunter-gatherers, Proc. Natl. Acad. Sci. USA 111, 728 (2014).

[20] V. Zaburdaev, S. Denisov, and J. Klafter, Lévy walks, Rev. Mod. Phys. 87, 483 (2015).

[21] R. N. Mantegna and H. E. Stanley, Stochastic Process with Ultraslow Convergence to a Gaussian: The Truncated Lévy Flight, Phys. Rev. Lett. 73, 2946 (1994).

[22] A. Miron, Universality in the Onset of Superdiffusion in Lévy Walks, Phys. Rev. Lett. 124, 140601 (2020). 
[23] Not to be confused with the term phase transition, that is typically used in the context of critical phenomena.

[24] A. Miron, J. Cividini, A. Kundu, and D. Mukamel, Derivation of fluctuating hydrodynamics and crossover from diffusive to anomalous transport in a hard-particle gas, Phys. Rev. E 99, 012124 (2019).

[25] H. Spohn, Nonlinear fluctuating hydrodynamics for anharmonic chains, J. Stat. Phys. 154, 1191 (2014).

[26] C. Godreche and J. M. Luck, Statistics of the occupation time of renewal processes, J. Stat. Phys. 104, 489 (2001).

[27] W. Wang, J. H. P. Schulz, W. Deng, and E. Barkai, Renewal theory with fat-tailed distributed sojourn times: Typical versus rare, Phys. Rev. E 98, 042139 (2018).

[28] S. Bianco, P. Grigolini, and P. Paradisi, Fluorescence intermittency in blinking quantum dots: Renewal or slow modulation? J. Chem. Phys. 123, 174704 (2005).

[29] Y. Edery, S. Berg, and D. Weitz, Surfactant Variations in Porous Media Localize Capillary Instabilities During Haines Jumps, Phys. Rev. Lett. 120, 028005 (2018).

[30] A. Weron, K. Burnecki, E. J. Akin, L. Sole, M. Balcerek, M. M. Tamkun, and D. Krapf, Ergodicity breaking on the neuronal surface emerges from random switching between diffusive states, Sci. Rep. 7, 5404 (2017).

[31] J. Klafter, A. Blumen, and M. F. Shlesinger, Stochastic pathway to anomalous diffusion, Phys. Rev. A 35, 3081 (1987).

[32] Note that Eq. (3) for $P_{n}(x, t)$ does not require an initial condition beyond that provided in Eq. (2) for $v_{n}(x, t)$.

[33] See Supplemental Material at http://link.aps.org/supplemental/ 10.1103/PhysRevResearch.2.032042 for a direct derivation of $\tilde{Q}_{n}(s)$, additional choices of the walk-time distribution, and detailed calculations.
[34] S. Benhamou, How many animals really do the Lévy walk? Ecology 88, 1962 (2007).

[35] D. W. Sims, D. Righton, and J. W. Pitchford, Minimizing errors in identifying Lévy flight behaviour of organisms, J. Anim. Ecol. 76, 222 (2007).

[36] M. C. Gonzalez, C. A. Hidalgo, and A.-L. Barabasi, Understanding individual human mobility patterns, Nature (London) 453, 779 (2008).

[37] T. H. Harris, E. J. Banigan, D. A. Christian, C. Konradt, E. D. T. Wojno, K. Norose, E. H. Wilson, B. John, W. Weninger, A. D. Luster et al., Generalized Lévy walks and the role of chemokines in migration of effector $\mathrm{CD}^{+} \mathrm{T}$ cells, Nature (London) 486, 545 (2012).

[38] A. Rebenshtok, S. Denisov, P. Hänggi, and E. Barkai, NonNormalizable Densities in Strong Anomalous Diffusion: Beyond the Central Limit Theorem, Phys. Rev. Lett. 112, 110601 (2014).

[39] N. Hazut, S. Medalion, D. A. Kessler, and E. Barkai, Fractional Edgeworth expansion: Corrections to the Gaussian-Lévy central-limit theorem, Phys. Rev. E 91, 052124 (2015).

[40] L. Zarfaty, A. Peletskyi, E. Barkai, and S. Denisov, Infinite horizon billiards: Transport at the border between Gauss and Lévy universality classes, Phys. Rev. E 100, 042140 (2019).

[41] U. Agrawal, S. Gopalakrishnan, R. Vasseur, and B. Ware, Anomalous low-frequency conductivity in easy-plane XXZ spin chains, Phys. Rev. B 101, 224415 (2020).

[42] D. A. Kessler and E. Barkai, Infinite Covariant Density for Diffusion in Logarithmic Potentials and Optical Lattices, Phys. Rev. Lett. 105, 120602 (2010).

[43] A. Dechant, E. Lutz, E. Barkai, and D. A. Kessler, Solution of the Fokker-Planck equation with a logarithmic potential, J. Stat. Phys. 145, 1524 (2011). 